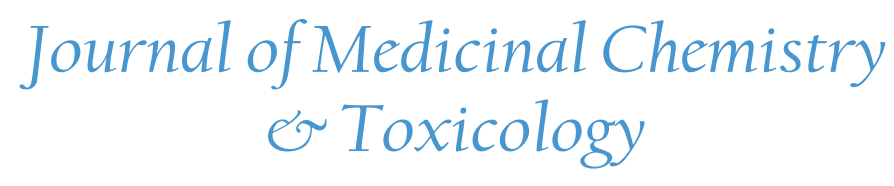

\title{
Effect of Steroidal Saponins-Loaded Composite Scaffolds on Bone Healing
}

\author{
Teklit Gebregiorgis Amabye*
}

Department of Chemistry, Mekelle University College of Natural and Computational Science, Ethiopia

*Corresponding authors: Teklit Gebregiorgis Amabye, Department of Chemistry, Mekelle University College of Natural and Computational Science, Ethiopia; E-mail: teklitgeb@gmail.com

\begin{abstract}
The objective of this study was to investigate the therapeutic potential of nano-bioglass/phosphatidylserine/collagen scaffolds loaded with steroidal saponins as an inducer factor for skeletal defects. The drugs-encapsulated bone substitute was prepared by loading steroidal saponins-collagen microsphere suspension in nano-bioglass and phosphatidylserine composite. The scaffolds possess an interconnected porous structure with a porosity of about $82.3 \%$. The pore size ranges from several micrometers up to about $400 \mu \mathrm{m}$. The drug release assays showed the long-term sustained release of steroidal saponins from the scaffolds with effective and safe bioactivity. Moreover, in vitro and in vivo studies showed that the involvement of steroidal saponins contributed to the secretion of nerve growth factor in MC3T3-E1 cells, which may be the possible factor that greatly enhanced bone healing. The results suggest that the bone substitute is an effective implantable drug-delivery system for use in bone repair.
\end{abstract}

Keywords: Steroidal saponins; Scaffold; Sustained release; Bone tissue engineering
Received Date: August 27, 2016

Accepted Date: August 30, 2016

Published Date: Sept 8, 2016

Citation: Amabye, T.G. Effect of Steroidal Saponins-Loaded Composite Scaffolds on Bone Healing. (2016) J Med Chem Tox 1(1): 18- 21.

DOI: $10.15436 / 2575-808 X .16 .1068$

\section{Introduction}

As the major pharmacological constituents of ophiopogon japonicus, a traditional Chinese medical herb, steroidal saponins are osteoinductive and can be used to treat bone and joint diseases ${ }^{[1]}$. However, steroidal saponins are usually not effective with oral administration because of enzymatic degradation and poor absorption. In addition, high concentration of steroidal saponins administered in the body was easy to cause coagulation reaction. Herein clinical use of the drug needs suitable system for its delivery, and this system should can release the drug in situ for appropriate time to interact with target cells at safe dose during bone healing ${ }^{[2]}$. In recent years, biodegradable scaffolds have been widely studied and become well established controlled drug delivery systems ${ }^{[3,4]}$. The forms are convenient because the slow releasing of drugs is preferred to sudden exposure of inducing factor ${ }^{[5]}$.

In our previous work, a biodegradable bone material, nano-hydroxyapatite/phosphatidylserine/collagen, was developed. In vitro and in vivo studies have indicated that the scaffold has good biodegradablility and osteoconductivity and promotes early mineralization and new bone formation ${ }^{[6]}$.

Beside that, we previously developed a novel collagen microencapsulation technology entrapping steroidal saponins in a self-assembled collagen matrix consisting of collagen fiber meshwork. These stable microspheres provide a physiologically relevant microenvironment to support osteoblast proliferation, viability, and differentiation ${ }^{[7]}$. Therefore the possibility of using steroidal saponins as an inducer factor assures better biological performance for bone regeneration.

Based on the fact that $\mathrm{nBG} / \mathrm{PS} / \mathrm{COL}$ scaffolds can enhance bone growth, the overall aim of this research was to confirm if bone substitute fashioned from collagen microspheres loaded with steroidal saponins and distributed in nBG/PS composite would produce more new bone than non-drug-loaded implants. To accomplish this aim, the steroidal saponins-loaded scaffolds were 
constructed, and the microstructure, drug release assays, and in vitro and in vivo biocompatible properties were evaluated and discussed.

\section{Materials and Methods}

Materials: 58s-BG was synthesized using a sol-gel method and consisted of $60 \mathrm{wt} \% \mathrm{SiO}_{2}, 35 \mathrm{wt} \% \mathrm{CaO}$ and $5 \mathrm{wt} \%$ $\mathrm{P}_{2} \mathrm{O}_{5}$. PS and NuSieve GTG agarose gel were supplied by Boao Medical Ltd. (Shanghai, China) and Liyuan Medical Ltd. (Xiamen, China), respectively. Lyophilized steroidal saponins were obtained from the College of Pharmacology, Shandong University of Traditional Chinese Medicine (Jinan, China).

Dulbecco's minimum essential medium (DMEM) and fetal bovine serum (FBS) were purchased from Sigma (St Louis, MO, USA). Tissue-culture clusters and flasks were purchased from Corning (Midland, MI, USA). All other reagents were of analytical grade.

Preparation of composite scaffolds: The steroidal saponins-loaded collagen microsphere was prepared as previously reported $^{[7]}$. The steroidal saponins-loaded nBG/PS/COL scaffolds were made with similar procedure of $\mathrm{nBG} / \mathrm{PS} / \mathrm{COL}$ composite $^{[6]}$. In brief, the $\mathrm{nBG}$ particles and drug-encapsulated collagen microspheres were gradually added to the solution of PS and intensively mixed. Then 1-ethyl-3-(3-dimethyl aminopropyl) carbodiimide (EDC) and N-hydroxysuccinimide (NHS) (4:1 ratio) were incorporated in deionized water at a content of 2.5 $\mathrm{mg} / \mathrm{mL}$ and the $\mathrm{pH}$ was adjusted to 5.5 with 2-(4-Morpholino) ethanesulfonic acid (MES); the solution was kept at $4^{\circ} \mathrm{C}$ for 24 $\mathrm{h}$ to allow cross-linking. The mixture was then transferred to a freezer (VXE380, Jouran, France) at $60^{\circ} \mathrm{C}$ for $12 \mathrm{~h}$ to solidify the solvent and induce solid-liquid phase-separation. The solidified mixture was freeze-dried in a freeze-dryer (ALPHA2-4, Christ, Germany) at $30^{\circ} \mathrm{C}$ for $24 \mathrm{~h}$ to form porous scaffolds. Control samples (nBG/COL/PS) were also prepared using the same process without the addition of steroidal saponins.

Microstructure observation: Scanning Electron Microscopy (SEM) (S-3400N, Hitachi Co., Tokyo, Japan) were used to investigate the microstructure of two groups of composite samples. The samples were sputter-coated with gold film for examination at the voltage of $20 \mathrm{kV}$.

In vitro drug release study: Drug release was determined by incubating $20 \mathrm{mg}$ of steroidal saponins-loaded nBG/PS/COL scaffolds or control cylinders $(10 \mathrm{~mm}$ in diameter and $20 \mathrm{~mm}$ in height) in $30 \mathrm{~mL}$ of isotonic phosphate buffered saline (PBS) at $37^{\circ} \mathrm{C}$. At $1,2,4,6,8,12,16$, and 40 days, the samples were centrifuged, and the supernatant was collected. The remaining wet materials were weighed and re-suspended in fresh PBS. The drug content of supernatant collected for each interval was determined by using the ultra performance liquid chromatography (UPLC, Waters Co., Milford, USA), and steroidal saponins were used as the standard.

The degradation ratio of steroidal saponins-loaded nBG/PS/COL scaffolds in PBS after 20 days of incubation was calculated by the formula of $\eta=(w 1-w 2) / w 1 \times 100 \%$. w1 and $w 2$ are respectively the weight of the composite before and after incubation.
Cell culture using scaffolds as supports: MC3T3-E1 cells (Japan Riken Cell Collection) were used to study the osteoblast biocompatibility of the two types of scaffolds in bone formation. Prior to the cell-culture assays, samples were sterilized by exposure to $\gamma$-irradiation. The cell density was adjusted to 1.0 $\times 10^{4}$ cells $/ \mathrm{mL}$, and $2 \mathrm{~mL}$ of cell suspension were seeded on the samples. After $24 \mathrm{~h}$ incubation, samples were washed with PBS and immersed in PBS containing 2.5\% glutaraldehyde ( $\mathrm{pH} 7.4)$ for $1 \mathrm{~h}$, then dehydrated through an ethanol series (from 30, 50, $70,90,95$ to $100 \%$ ), followed by critical-point dried and then coated with gold for SEM examination.

Reverse transcription-polymerase chain reaction (RT-PCR) analysis: RT-PCR was performed using standard methods, as previously described ${ }^{[8]}$. PCR products were electrophoresed on a 2\% NuSieve GTG agarose gel, stained with ethidium bromide, and detected with a fluorescence image analyzer (Cellometer Vision CBA, Nexcelom, MA, USA). Primers were designed as follows: NGF $\left(5^{\prime} \rightarrow 3^{\prime}\right)$ : Forward, TCCACCCACCCAGTCT-TC$\mathrm{CAC}$;

Reverse, GCCTCTTCTTGTAGC-CTTCCT.

In vivo biocompatibility evaluation: The reaction of bone-tissue to the scaffolds was examined using a rabbit tibia defect model. Two 20-mm bone defects were created in two groups of five rabbits. The right defect was an untreated control, whereas the left defect had one of two types of implants: (1) nBG/PS/ COL scaffolds, or (2) steroidal saponins-loaded nBG/PS/COL scaffolds.

The implanted composite and surrounding tissues were removed 8 weeks after operation. Samples were decalcified, embedded in paraffin and sectioned prior to histological staining using hematoxylin and eosin (HE) and observed using light microscopy.

\section{Results}

The SEM micrographs of the scaffolds with and without steroidal saponins are shown in Figure 1. Two types of scaffolds both possess interconnected porous structure, which indicates that the involvement of steroidal saponins did not significantly affect the porous morphologies. The porosity of steroidal saponins-loaded scaffolds $(82.3 \pm 4.8 \%)$ is also similar with that of steroidal saponins free scaffolds $(81.9 \pm 5.1 \%)$, and the pore size ranges from several micrometers up to about $400 \mu \mathrm{m}$.

\section{Microstructure of steroidal saponins-loaded scaffolds}

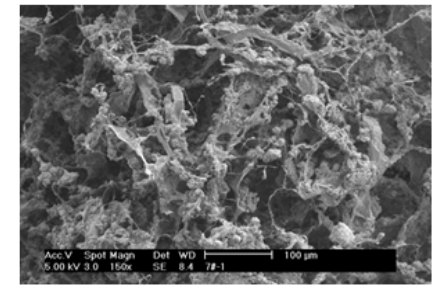

a

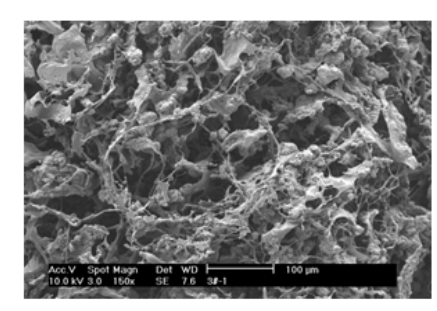

$\mathrm{b}$
Figure 1: SEM morphologies of (a) steroidal saponins-loaded nBG/PS/ COL scaffolds and (b) nBG/PS/COL scaffolds without steroidal saponins. 
Figure 2 shows steroidal saponin release from $\mathrm{nBG} / \mathrm{PS} /$ COL scaffolds. The profile shows a burst of drug $(=18 \%)$ on the first two day with a total of $60 \%$ to $70 \%$ of the loaded drug released from the scaffolds in 10 to 15 days, followed by slow release of bound drug starting at around 25 days. This result corresponds to our early release profiles reported for collagen microspheres $^{[7]}$.

\section{In vitro drug release}

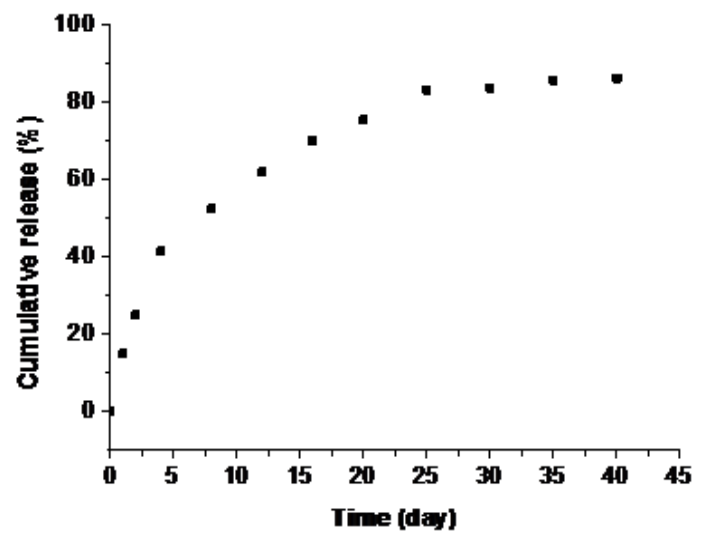

Figure 2: Release profile of steroidal saponins from nBG/PS/COL scaffolds over 40 days in vitro

Our previous study showed that the application of steroidal saponins to MC3T3-E1 cells enhanced the biomineralization and proliferation of cells ${ }^{[7]}$. But the reasons by which the administration of steroidal saponins improved bioactivity in this model are vague. In this report, when we analyzed the MC3T3-E1 cells cultured together two types of scaffolds by RT-PCR, cells expressed the classical neurotrophin, such as nerve growth factor (NGF) (Figure 3). Beyond that, the cells cultured together with scaffolds that contained steroidal saponin expressed significantly more NGF than either type of scaffolds without the drug.

\section{Modulation of neurotrophin expression in MC3T3-E1 cells.}
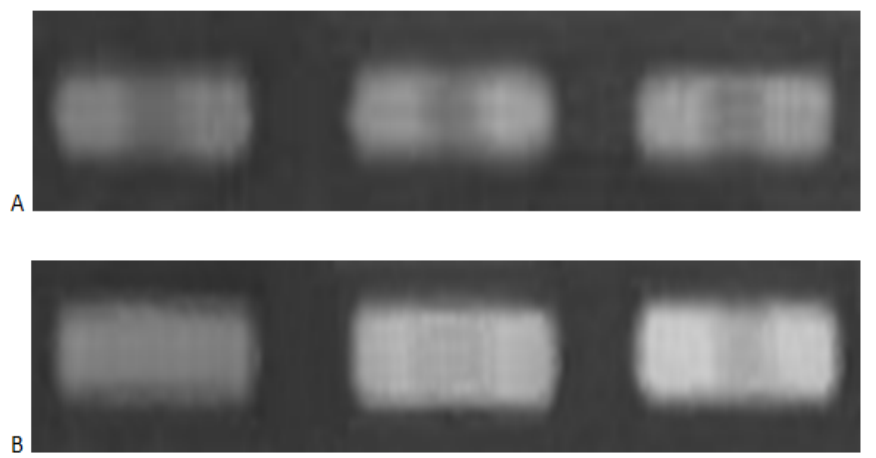

0

$12 \mathrm{~h}$

$24 \mathrm{~h}$

Figure 3: RT-PCR analysis of NGF mRNA expression in MC3T3-E1 cells cultured together with $\mathrm{nBG} / \mathrm{PS} / \mathrm{COL}$ scaffolds without steroidal saponins (a) and with steroidal saponins, (b) after $12 \mathrm{~h}$ and $24 \mathrm{~h}$, respectively.
In order to further evaluate the bioactivity of steroidal saponins-loaded $\mathrm{nBG} / \mathrm{PS} / \mathrm{COL}$ scaffolds, implant experiments in vivo were carried out. Figure 4 shows representative samples of the histology analysis of each treatment in the rabbit bone defect. Clearly, no treatment results in little healing, with only occasional islands of new bone emanating from the defect margins, a potential source of bone growth factors (Figure 4a). Treatment with implants (with no drugs) does a little to enhance healing. Islands of bone formed along the interface between the implants and local tissues, but a full table had not formed by week 8 (Figure $4 \mathrm{~b}$ ). However, defects treated with the steroidal saponins-loaded implants produced bone tables at the implant margins and the periosteum. The sum of new bone and type, and the amount of collagen present were significantly higher. The bone appeared to be maturing, tending toward remodeling with lamellar bone (Figure 4c).

\section{In vivo biocompatibility evaluation:}
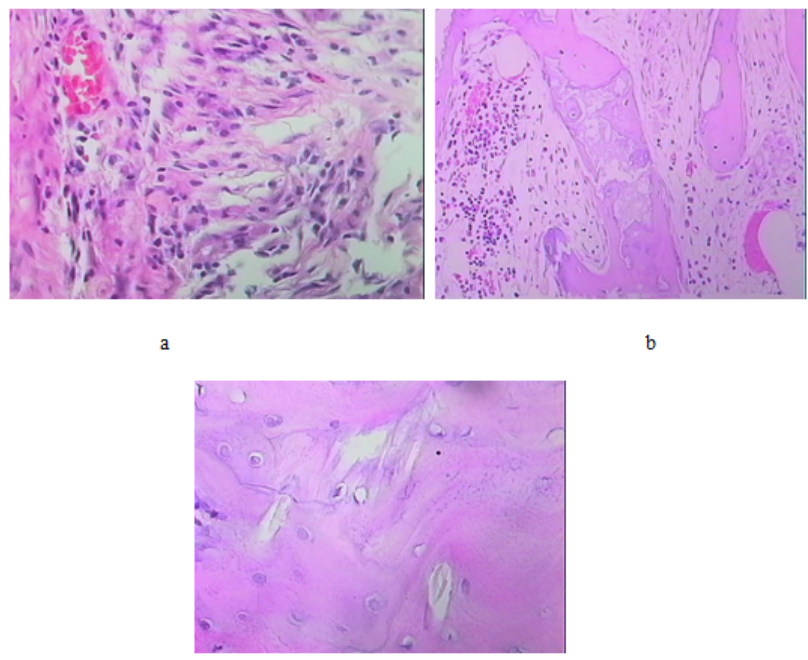

Figure 4: Histological morphology (HE stain magnification $\times 40$ ) of bone defects 8 weeks after implantation. (a) Untreated; (b) nBG/PS/ COL scaffolds without steroidal saponins; (c) steroidal saponins-loaded nBG/PS/COL scaffolds.

\section{Discussion}

Steroidal saponin has already been used for bone and joint diseases, nerve damage, and hematischesis. The therapeutic drug concentration must be controlled by drug monitoring. Previous study showed that high plasma concentrations of steroidal saponin will cause coagulation reaction ${ }^{[1]}$. Therefore, less than $12 \mu \mathrm{g} / \mathrm{ml}$ plasma concentration was suggested in order to prevent the side effects.

In our previous study, the $\mathrm{nBG} / \mathrm{PS} / \mathrm{COL}$ bone substitute has been proven to have unique osteoconducntion and osteoinduction ${ }^{[6]}$. The appropriate degradation characteristics and porous microstructure make it possible to control the kinetic property of drug release forming a safe and effective drug concentration in local tissues. Further, the scaffolds had sustained release of drugs in 40 days that gave the implants a long-term activity.

Makio et al had reported that NGF is essential for osteoblastic cell survival as an autocrine anti-apoptotic factor ${ }^{[9]}$. The present study has showed that the released steroidal saponins can specifically initiate the secretion of NGF in MC3T3-E1 
cells. NGF plays an important role as counterbalance and a counterpart to the apoptosis of the osteoblastic cells, and will affect the life span of cells ${ }^{[10]}$. Additionally, NGF was detected in osteoblasts, which suggests that the osteoblasts may govern innervation by synthesizing and secreting $N \mathrm{NF}^{[11]}$. Sympathetic and sensory nerve fibers mainly present in the periosteum ${ }^{[12]}$. Therefore the form of NGF in osteoprogenitor cells should be effective to the normal preservation of nerve fibers. On the other hand, similar embryonal-like skeletal cells are mainly found in the initial stage of fracture callus, therefore cells of the callus should similarly contain NGF. In the present study NGF presence by cellular components has been presented as a possible reason which increases this callus.

\section{Conclusion}

A bone graft substitute was prepared by loading steroidal saponins/collagen microsphere suspension in our previously developed biomimetic nBG-PS composite. The drug release assays showed the long-term sustained release of steroidal saponins from the $\mathrm{nBG} / \mathrm{PS} / \mathrm{COL}$ composite with safe and effective bioactivity. And the involvement of steroidal saponins contributed to the secretion of NGF in MC3T3-E1 cells, which may be the possible factor that greatly enhanced bone healing. Therefore, it is reasonable to hypothesize that such bone substitute could be an effective implantable drug-delivery system for use in bone repair

\section{References}

[1] Wang, Y. Z., Wang, J.J., Liang, J.C., et al. Effects of diosgenin on cell proliferation, differentiation and OPG/RANKL mRNA expression of rat osteoblasts cultured in vitro. (2010) China J Tra Chinese Med Pharm 25(1): 134-136.

[2] Lucas, P.A., Syftestad, G.T., Goldberg, V.M., et al. Ectopic induction of cartilage and bone by water soluble proteins from bovine bone using a collagenous delivery vehicle. (1989) J Biomed Mater Res 23: 23-39.

[3] Kang, B.J., Kim, Y., Lee, S., et al. Collagen I gel promotes omogenous osteogenic differentiation of adipose tissue-derived mesenchymal stem cells in serum-derived albumin scaffold. (2013) J Biomater Sci Polym Ed 24(10): 1233-1243.

[4] Goel, S.C., Singh, D., Rastogi, A., et al. Role of tricalcium phosphate implant in bridging the large osteoperiosteal gaps in rabbits. (2013) Indian J Exp Biol 51(5): 375-380.

[5] Nilsson, M., Zheng, M.H., Tägil, M. The composite of hydroxyapatite and calcium sulphate: a review of preclinical evaluation and clinical applications. (2013) Expert Rev Med Devices 10(5): 675-684.

[6] Yang, C.R., Wang, Y.J, Chen, X.F. Mineralization regulation and biological influence of bioactive glass-collagen-phosphatidylserine composite scaffolds. (2012) Sci China Life Sci 55(3): 236-240.

[7] Yang, C.R., Cong, Fang. Microporous nano-hydroxyapatite/collagen/phosphatidylserine scaffolds embedding collagen microparticles for controlled drug delivery in bone tissue engineering. (2015) Mat Res 18(5): 1077-1081.

[8] Cohen, G., Ettinger, K., Lecht, S., et al. Transcriptional Down- regulation of Epidermal Growth Factor (EGF) Receptors by Nerve Growth Factor (NGF) in PC12 Cells. (2014) J Mol Neurosci 54(3): 574-585.

[9] Mogi, M., Kondo, A., Kinpara, K., et al. Anti-apoptotic action of nerve growth factor in mouse osteoblastic cell line. (2000) Life Sci 67(10): 1197-1206.

[10] Grills, B.L, Schuijers, J.A., Ward, A.R. Topical application of nerve growth factor improves fracture healing in rats. (1997) J Orthop Res 15(2): 235-242.

[11] Nakanishi, T., Ohyama, K., Aoki, C., et al. Expression of trkC in a mouse osteoblastic cell line and its response to neurotrophin-3. (1994) Bioc Biophys Res Commun 203(2): 1268-1274.

[12] Grills, B.L., Schuijers, J.A. Immunohistochemical localization of nerve growth factor in fractured and unfractured rat bone. (1998) Acta Orthop Scand 69(4): 415-419.
Ommega Online Publisher

Journal of Medicinal Chemistry \& Toxicology

Short Title : J Med Chem Toxicol
ISSN No: 2575-808X

E-mail: medchemtoxic@ommegaonline.org

website: www.ommegaonline.org 